\title{
Development of a 3.5-inch Magneto-Optical Disk with a Capacity of 2.3 GB
}

\author{
Mineo Moribe, Keiji Shono, Miyozo Maeda ${ }^{*}$,Koichi Ogawa ${ }^{*}$, Hiroshi Nakayama ${ }^{* *}$ and Mikio Yoshida ${ }^{* *}$ \\ Storage Systems Laboratories, Fujitsu Laboratories Limited, 64 Nishiwaki, Okubo-cho, Akashi 674-8555 \\ * Optical Disk Systems Division, Fujitsu Limited, 4-1-1 Kamikodanaka, Nakahara-ku, Kawasaki 211-8588 \\ **Disk Media Department, CNC RM Company, Sony Corporation, 3-4-1 Sakuragi, Tagajo, 985-0842
}

The recording capacity of GIGAMO media was enlarged from 1.3 GB to $2.3 \mathrm{~GB}$ for 3.5 inch Magneto-Optical (MO) disks while maintaining downward compatibility. For the new GIGAMO technology, a land and groove recording method was applied in addition to magnetically induced super resolution (MSR) media. Furthermore, a novel address format suitable for the land and groove recording method was adopted. The specifications of the new GIGAMO media were examined to satisfy requirements for practical use with respect to margins. Durability of more than $10^{6}$ rewritings and an enough lifetime were confirmed.

Key words: magneto-optical recording, MSR, land and groove recording, address format, compatibility

\section{Introduction}

By using magnetically induced super resolution (MSR) technology, 3.5 inch Magneto-Optical (MO) disks with 1.3-GB capacity named GIGAMO were commercialized in $1998{ }^{1)}$. A conventional optical system equipped with a $685-\mathrm{nm}$ wavelength laser diode and an objective lens with a 0.55 numerical aperture were used in the GIGAMO drive. Furthermore, an on-land recording method was adopted to maintain downward compatibility with all ISO-standardized disks with capacities from $128 \mathrm{MB}$ to $640 \mathrm{MB}$. The MSR media used for GIGAMO is called Double-mask Rear Aperture Detection (D-RAD).

The D-RAD media features two magnetic masks that are formed on the media due to the temperature distribution resulting from laser beam irradiation ${ }^{2}$. This D-RAD media enables higher resolution not only in the tangential direction but also in the radial direction. Consequently, crosstalk signal from adjacent tracks can be reduced, making land and groove recording for increased capacity easier. The possibility of achieving a $2.4 \mathrm{~GB}$ capacity per single-side of a 3.5 inch disk by combining D-RAD media and the land and groove recording was previously reported ${ }^{3}$. In this paper, practical technologies to enlarge the capacity of GIGAMO from $1.3 \mathrm{~GB}$ to $2.3 \mathrm{~GB}$ with a slight change in the wavelength of the laser diode, while maintaining complete downward compatibility, are described.

\section{Disk Structure}

\section{2-1 Substrate and MSR media}

Molded polycarbonate substrates with land and groove structure are prepared. Land and groove structure features both track pitch and groove depth. In this investigation, various track pitch media are prepared and the optimum track pitch is determined to be $0.67 \mu \mathrm{m}$ by the process described in the next section. Groove depth is determined by considering MO signal quality and ID signal quality. In general, shallow groove gives high reflectivity and high MO carrier level, but low ID pit signal amplitude. In this investigation, groove depth is fixed to
$45 \mathrm{~nm}$ for balancing both signal properties.

The D-RAD media are fabricated as shown in Fig 1. They are essentially the same as those of 1.3 GB GIGAMO media.

\begin{tabular}{cc}
\hline \multicolumn{3}{c}{ Substrate (PC) } \\
\hline Dielectric Layer & $\mathrm{SiN}$ \\
\hline Readout Layer & $\mathrm{GdFeCo}$ \\
\hline Intermediate Layer & $\mathrm{GdFe}$ \\
\hline Recording L Lyer & $\mathrm{TGFeC}$ \\
\hline Dielectric Layer & $\mathrm{SiN}$ \\
\hline Heat-sink & $\mathrm{Al}$ \\
\hline
\end{tabular}

Fig 1 Cross section of new GIGAMO disk.

\section{2-2 Address format}

A 3.5-inch MO disk is divided into plural recording sectors, and their address information is important for quick access to target sectors. Conventional MO disks, including GIGAMO disks and ISO-standardized disks, have header (ID) signals generated from embossed pits at the top of sectors. In the new GIGAMO disks, ID signals are also placed at the top of sectors to make compatibility easier. To obtain large ID signal amplitude, a narrower groove in the ID area is desirable. On the other hand, a wider groove gives larger tracking signal. Therefore, for a range in which the tracking signal is acceptable, the groove width in the ID area must be narrower than that in the MO recording area. As the embossed pits cannot be detected by using the MSR method, the shortest pit length must be set longer than the shortest MO mark length. As a result, two clock frequencies, one for ID signal reading and the other for MO signal processing, are required in the MO drive. To produce two clock signals easily, pit lengths also have to be exact multiples of the MO mark length. Pit lengths are set three times as long as MO marks to obtain a sufficient ID signal. Furthermore, as the ID signal from neighboring tracks tend to cause crosstalk, the ID pits for land tracks and those for groove tracks are shifted toward each other in the tangential direction, as shown in Fig. 2. This technique is named Stagger ID. In this structure, vfo signal (the shortest marks and spaces) amplitude normalized by the reflection amplitude of the data area is more than 0.15 . This value is large enough for stable ID reading. The Stagger ID is superior in terms of compatibility. However, as the header areas are long, the data efficiency is reduced. Therefore, we improved the format such that one header for two sectors is placed alternately on the land track and groove track as shown in Fig. 2(b). Consequently, both compatibility and a data efficiency as high as $74 \%$ are obtained. 


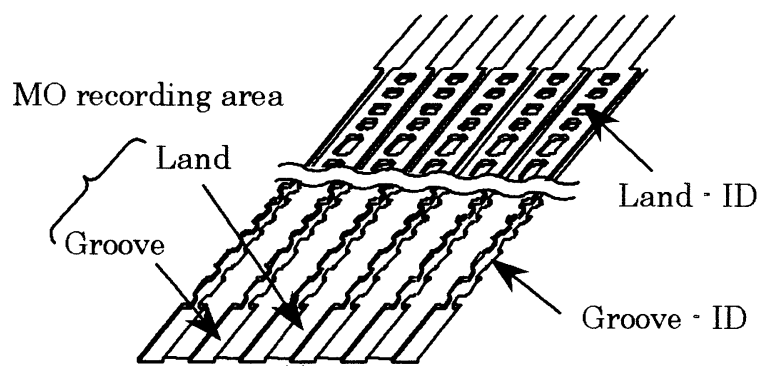

(a) Surface structure

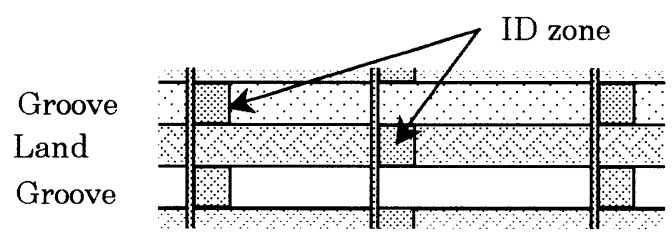

(b) Arrangement of ID zones

Fig. 2 Structure of Stagger ID

\section{Evaluation method for write/read properties}

\section{3-1 Testing instruments}

Acommercially available MO tester (Pulstec DDU-1000: NA $=0.55, \lambda=660 \mathrm{~nm})$ and a newly developed GIGAMO drive ( $\mathrm{NA}=0.55, \lambda=665 \mathrm{~nm}$ ) are used to evaluate write/read properties of disks and specifications of the media are determined. In the MO tester measurement, linear velocity and the shortest mark length are set to $7.5 \mathrm{~m} / \mathrm{s}$ and $0.3 \mu$ $\mathrm{m}$, respectively. In the drive measurement, parameters other than those to be tested are automatically optimized to minimize bit error rate. Both in the $\mathrm{MO}$ tester measurement and the drive measurement, no special signal processing method such as PRML is applied.

\section{3-2 Crosstalk}

In ordinary optical disks, crosstalk increases in proportion to read power. On the other hand, D-RAD media shows peculiar crosstalk behavior as is shown in Fig. 3 (b). With increasing read power, crosstalk is maintained at a constant level until about $5 \mathrm{~mW}$ and then crosstalk increases catastrophically. Crosstalk at lower read powers is small enough. However, the sudden increase of crosstalk at higher read powers is not acceptable and the starting point of the increase depends mainly on track pitch. Crosserase doesn't show such a catastrophic increase with increasing erase power. Thus, crosstalk is precisely evaluated according to the following three steps using the MO tester.

i) A 2T-pattern signal of $(1,7)$ RLL code is written on a center track at optimum write power, which gives CNR maximum. Then, the signal is read at optimum read power and at the readout magnetic field, and its carrier level is measured. In this measurement, the optimum read power is a power, which gives the maximum CNR and is set to $30 \%$ higher power than the lowest read power at which signal can be detected.

ii) The signal on the center track is erased, and the 2T-pattern is written on the neighboring tracks at optimum write power, at $4 \%$ higher than optimum power, and at $8 \%$ higher than optimum power. This last value is almost the same as the maximum power that can be controlled by the firmware in the new GIGAMO drives iii) The center track signal is read at the optimum read power and readout field, and its carrier level is measured. The difference of the carrier levels of step i) and step iii) is defined as crosstalk.

Crosstalk is also evaluated while varying disk tilt and tracking offset. In this measurement, 8Tpattern signals are written on the neighboring tracks with optimum power and without disk tilt and track offset, and the erased center track is read with disk tilt and track offset.

\section{3-3 Read and write power margins}

Several types of margin for practical use are examined. First, the read power margin and the write power margin are examined. These margins are evaluated by using a new GIGAMO drive. Random pattern signal is written and the bit error rate is measured. Margin maps are made while varying read power and readout field.

3-4 Durability

Durability tests with a cyclic erase/write/read and an accelerated lifetime test are conducted.

To use a disk as computer memory requires, that its properties remain unchanged after cyclic erase/write/read use. Thus, cyclic tests are performed by using a new GIGAMO drive at 55 degrees Celsius condition, which is the highest temperature of operating environment in the ISO standard for MO media. The bit error rates before and after $10^{6}$ cycles are compared to evaluate the media's durability.

In general, MO media are well known to be long-life media. Since GIGAMO media have similar structure and materials as those of ordinary MO media, their long-life can be anticipated. In the lifetime test, the new GIGAMO media are placed in an oven at 80 degree Celsius and $85 \%$ relative humidity, and the bit error rates are measured periodically.

\section{Results and discussions}

\section{4-1 Determination of specifications}

Under narrow track pitch land and groove conditions, crosstalk can take place even in D-RAD media. Crosstalk for several types of D-RAD media with a track pitch of $0.65 \mu \mathrm{m}$ and groove depth of $45 \mathrm{~nm}$ is investigated, where the readout magnetic field is $400 \mathrm{Oe}$. The results for two typical types of media are shown in Fig. 3, where Gd content in the GdFeCo readout layer is varied. Fig. 3 shows low Gd content media (a) and high Gd content media (b), and their cross talk carrier levels plotted with changing read power. The three lines in each graph correspond to the carrier levels when marks on the neighboring tracks are written at optimum power ( $\mathrm{Pw}$ opt), at power $4 \%$ higher than the optimum $(\mathrm{Pw}+4 \%)$, and at power $8 \%$ higher than the optimum ( $\mathrm{Pw}+8 \%)$, respectively. 'The criterion of acceptable crosstalk is set to $-23 \mathrm{~dB}$. When the Gd content of the readout layer is low, crosstalk gradually increases with increasing read power and easily exceeds the acceptable level. On the contrary, crosstalk remains at a constant level between 3.5 and $5 \mathrm{~mW}$ when the Gd content of the readout layer is high. Excessively high read power over 5 $\mathrm{mW}$ raises the temperature of neighboring tracks and the front mask breaks down.

Regarding why high Gd content in the readout layer of media has the effect of suppressing crosstalk, a reason in terms of the front mask formation field can be offered ${ }^{4}$. A front mask formation field is defined as a 
magnetic field required for formation of a front mask during readout operation. The front mask formation field is measured with varying write powers on neighboring tracks. As is shown in Fig. 4, for media having low $\mathrm{Gd}$ content in the readout layer, the readout magnetic field required for formation of the front mask

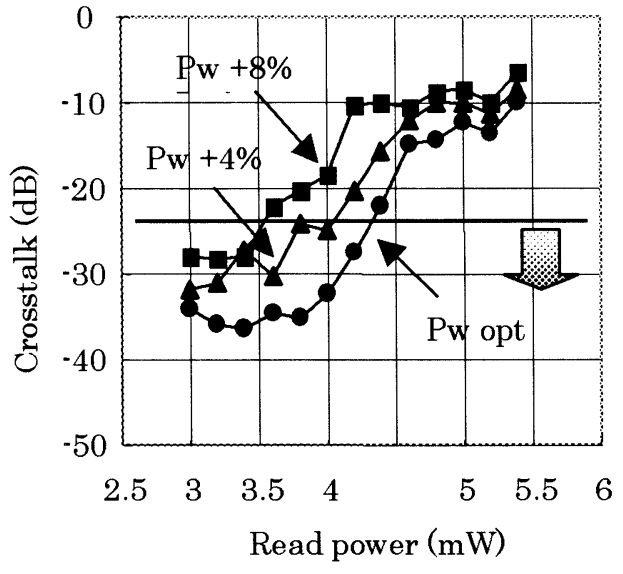

(a) Low Gd content

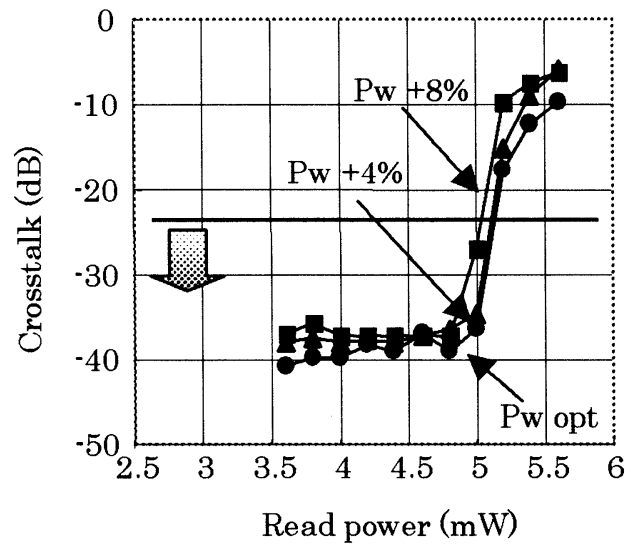

(b) High Gd content

Fig. 3 Read power dependence of crosstalk for low and high Gd content media. Mark length is $0.3 \mu \mathrm{m}$.

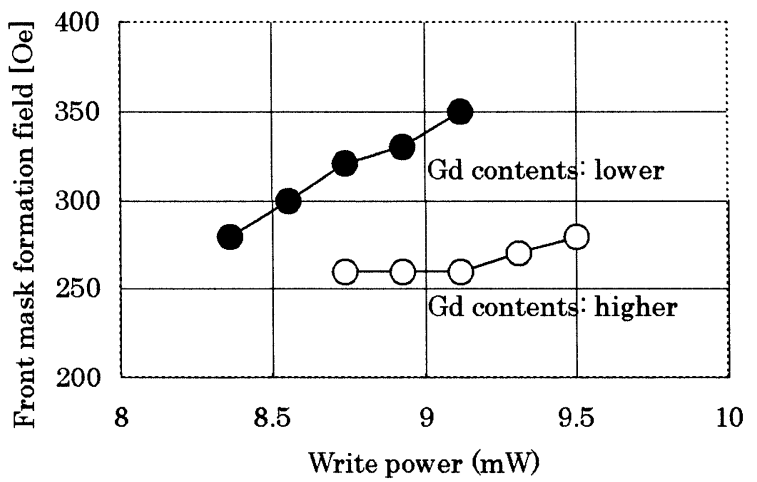

Fig. 4 Write power dependence of front mask formation field for media with low Gd content (black circles) and with high Gd content (white circles) in readout layer. increases as the write power on neighboring tracks increases. In this case, the front mask does not form if the readout magnetic field is insufficient. Specifically, in the "low Gd" media, front mask formation is made difficult by strongly written marks, and that causes significant crosstalk. On the other hand, when Gd content in the readout layer is high, the magnitude of the front mask formation field remains low and the magnitude of the front mask formation field remains low and the readout magnetic field is sufficient. As is discussed in the previous paper ${ }^{4)}$, the front mask is formed in the following way (see Fig. $5^{4)}$ ). The magnetic direction of the intermediate layer is aligned parallel to the readout magnetic field, which is applied parallel to the writing direction. Consequently, the magnetic direction of the readout layer is aligned anti-parallel to the magnetic direction of the intermediate layer, because the readout layer and intermediate layer are exchange coupled at low temperature. The magnetization of the intermediate layer is larger than that of the readout layer at low temperature. Considering this, smaller magnetization of the readout layer makes the front mask formation easier. High Gd content makes the magnetization of the readout layer small, resulting in reduction of the front mask formation field. However, too much Gd content leads to poor MO signal quality. Consequently, Gd content in the readout layer must be optimized carefully.

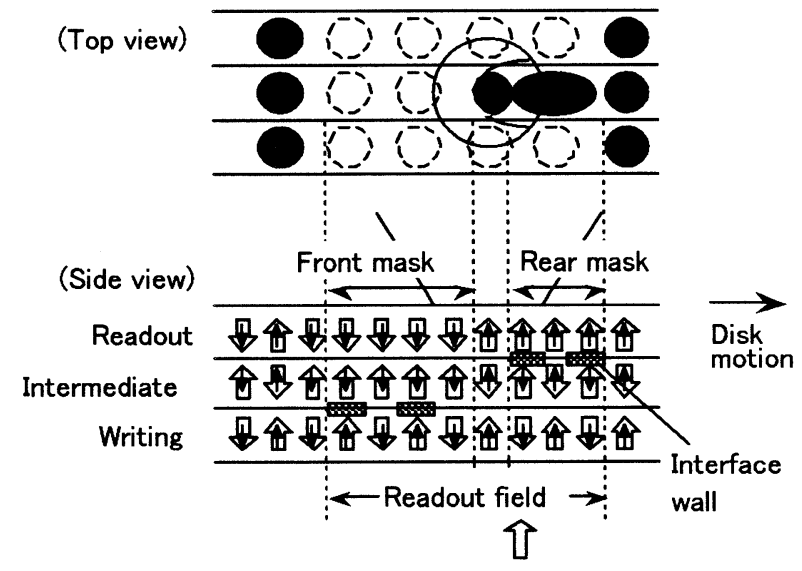

Fig. 5 Illustration of magnetization configuration during readout with readout field in writing direction.

Track pitch is determined by considering disk tilt and detracking as well as crosstalk. Crosstalk is measured while detracking, and a detrack margin is obtained that is defined as the maximum detrack range where an acceptable crosstalk signal, less than $-23 \mathrm{~dB}$, is observed. Fig. 6 (a) and (b) shows the detrack margin, with the form of \pm for $0.6 \mu \mathrm{m}$ track pitch (a) and $0.65 \mu \mathrm{m}$ track pitch (b), respectively, that are plotted while varying the disk tilt. The $G \rightarrow L$ and $L \rightarrow G$ in these figures represent the crosstalk from groove track to land track, and those from land track to groove track, respectively. For the new GIGAMO drive, a detrack margin greater than $\pm 0.08 \mu \mathrm{m}$ and a tilt-margin over $\pm 8 \mathrm{mrad}$ are required. Considering these values, it is concluded that a track pitch wider than $0.65 \mu \mathrm{m}$ is necessary. Taking other margins in disk production into account, track pitch as $0.67 \mu \mathrm{m}$ is finalized. 
The specifications of the new GIGAMO media are summarized in Table 1 and compared with those of conventional media. For downward compatibility, the new GIGAMO media have the same cartridge size and substrate thickness as those of conventional media. Mark length and track pitch can be remarkably shortened by using land and groove recording and MSR technology. Minimum bit length is determined as $0.23 \mu \mathrm{m}$ so that a bit error rate lower than $1 \times$ $10^{-5}$ is obtained assuming $(1,7)$ RLL code. Expressed in term of recording density, the new GIGAMO has a density of 4.2 Gbit/in².

Table 2 summarizes the specifications of the new GIGAMO drive for three types of the media. The data transfer rate of 8.38 $\mathrm{MB} / \mathrm{s}$ is achieved for the new GIGAMO media.

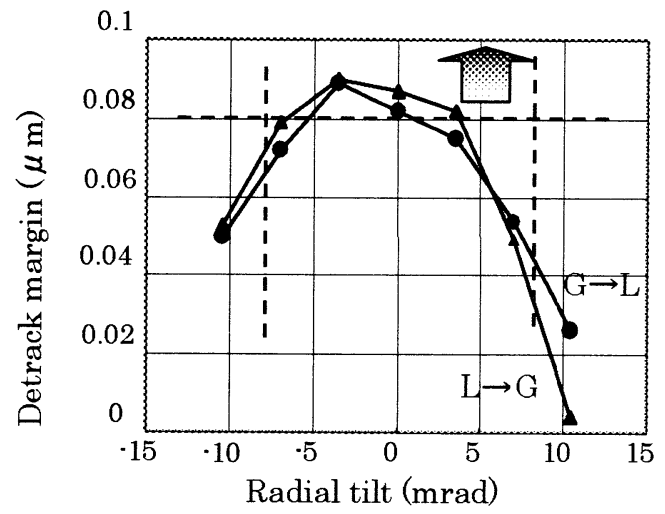

(a) Track pitch of $0.6 \mu \mathrm{m}$ media

\section{4-2 Read/Write power margins}

The write and read power margins of the new GIGAMO system are measured. Random pattern signal is written on three contiguous tracks and the bit error rate on the center track is measured, with varying read or write power in read or write power margin measurements, respectively. Results are shown in Fig. 7. A write power margin as wide as $13 \%$ p p is obtained for both the land and groove track when a bit error rate of 1 $\times 10^{-4}$ is acceptable. The read power margin is wider than $40 \%$ p.p for land and groove tracks. As the new GIGAMO drive has sophisticated test-write and test-read function, these power margins are sufficiently wide for practical use.

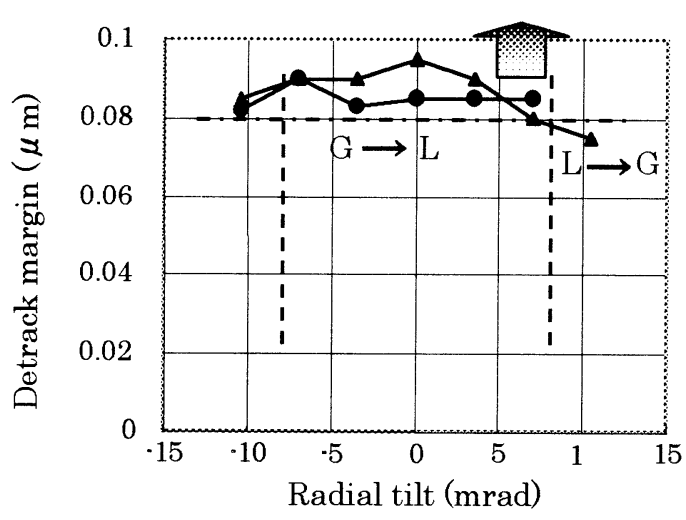

(b) Track pitch of $0.65 \mu \mathrm{m}$ media

Fig. 6 Detrack margin ( \pm ) derived from crosstalk less than $-23 \mathrm{~dB}$, as function of disk tilt. Mark length is $0.3 \mu \mathrm{m}$.

Table 1 Specifications of 3.5 inch MO media

\begin{tabular}{l|c|c|c}
\hline Standard & 2.3-GB GIGAMO & 1.3-GB GIGAMO & ISO/IEC 15041 \\
\hline $\begin{array}{l}\text { Memory capacity / } \\
\text { Cartridge size }\end{array}$ & $2.3 \mathrm{~GB}$ & $1.3 \mathrm{~GB}$ & $540 \& 640 \mathrm{MB}$ \\
\hline Cartridge size & $90 \times 94 \times 6(\mathrm{~mm})$ & $90 \times 94 \times 6(\mathrm{~mm})$ & $90 \times 94 \times 6(\mathrm{~mm})$ \\
\hline Substrate thickness & $1.2 \mathrm{~mm}$ & $1.2 \mathrm{~mm}$ & $1.2 \mathrm{~mm}$ \\
\hline Track pitch & $0.67 \mu \mathrm{m}$ & $0.90 \mu \mathrm{m}$ & $1.10 \mu \mathrm{m}$ \\
\hline Bit length of MO area & $0.23 \mu \mathrm{m}$ & $0.29 \mu \mathrm{m}$ & $0.49 \mu \mathrm{m}$ \\
\hline Linear density (ID) & 2,200 channel bits $/ \mathrm{mm}$ & $2,634 \mathrm{channel} \mathrm{bits} / \mathrm{mm}$ & $3,125 \mathrm{channel} \mathrm{bits} / \mathrm{mm}$ \\
\hline Linear density (MO) & 6,601 channel bits $/ \mathrm{mm}$ & $5,268 \mathrm{channel} \mathrm{bits} / \mathrm{mm}$ & $3,125 \mathrm{channel} \mathrm{bits} / \mathrm{mm}$ \\
\hline
\end{tabular}

Table 2 Specifications of new GIGAMO drive

\begin{tabular}{l|c|c|c}
\hline Item & \multicolumn{3}{|c}{ Specification } \\
\hline Media & $2.3 \mathrm{~GB}$ & $1.3 \mathrm{~GB}$ & $640 \mathrm{MB}$ \\
\hline Data transfer rate & Max. $8.38 \mathrm{MB} / \mathrm{s}$ & Max. $6.70 \mathrm{MB} / \mathrm{s}$ & Max. $5.87 \mathrm{MB} / \mathrm{s}$ \\
\hline Average seek time & \multicolumn{3}{|c}{$19 \mathrm{~ms}$ (Typ) $/ 20 \mathrm{~ms}$ (Max) } \\
\hline Disk rotation speed & $3,637 \mathrm{rpm}$ & $\begin{array}{c}3,637 \mathrm{rpm} \\
4,801 \mathrm{rpm}(\mathrm{ZCLV})\end{array}$ & $5,455 \mathrm{rpm}$ \\
\hline Host interface & \multicolumn{3}{|c}{ ATAPI, SCSI-2, USB2.0 } \\
\hline Electricity (average) & +5V DC $\pm 5 \%, 1.3 \mathrm{~A}(2.5 \mathrm{AMax}$ ) \\
\hline
\end{tabular}


Fig.8 (a) and Fig. 8 (b) show bit error rate maps as a function of readout magnetic field and read power, measured at room temperature (a) and 55 degrees Celsius (b). In each experiment, signals on neighboring tracks are written with an upper limit power ( $8 \%$ higher than optimum power) and the center tracks are reproduced with varying readout field and read power. The D-RAD function works by increasing the read power or the readout field, and the bit error rate abruptly improves.

Excessive increase in readout field or read power causes crosstalk and the bit error rate becomes worse. At 55 degree Celsius, the optimum centers with respect to read power and readout field shift about $0.4 \mathrm{~mW}$ and 50 Oe, respectively, compared with those measured at room temperature condition. However, since the new GIGAMO drive has a sophisticated firmware function for finding the optimum read and write conditions, these shifts due to temperature change cause no problems.

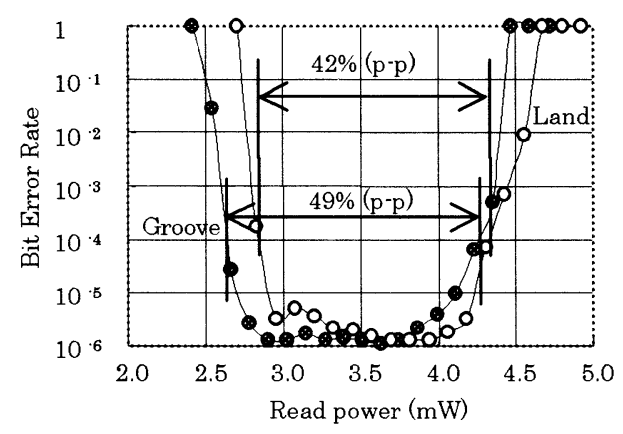

(a) Read power margin

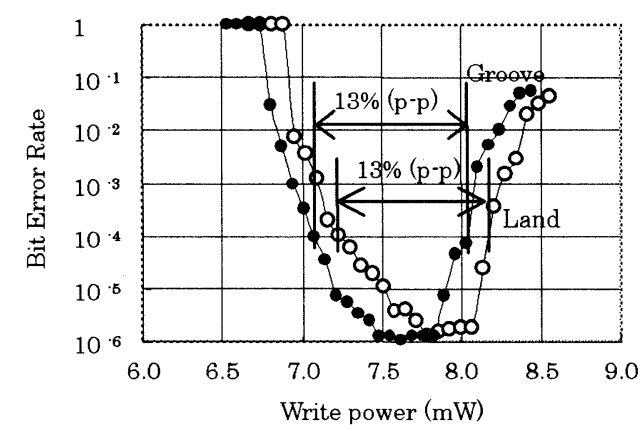

(b) Write power margin

Fig. 7 Power margins measured by using newly developed GIGAMO drive.

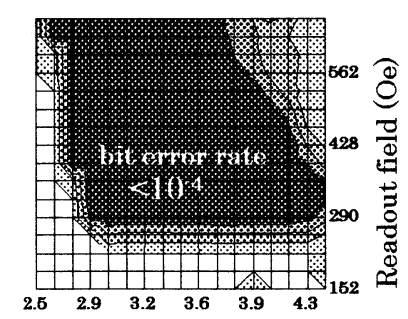

Read power $(\mathrm{mW})$

a) Room temperature

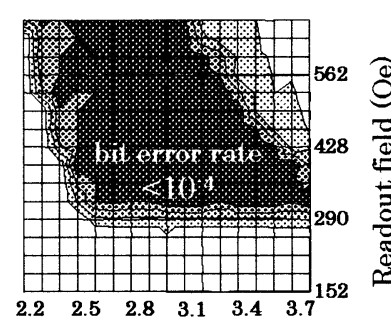

Read power $(\mathrm{mW})$

(b) $55^{\circ} \mathrm{C}$
Fig. 8 Bit error rate map as functions of read power and readout magnetic field.

\section{4-3 Durability}

Fig. 9 shows the results of a cyclic erase, write and read test conducted at 55 degrees Celsius. In this cycle, erase, write and read power are set at optimum values selected by the drive. Even after $10^{6}$ cycles, the change in the bit error rate is quite small. The change in power sensitivity is also small.

Fig. 10 shows the bit error rate change under the conditions of 80 degrees Celsius and $85 \%$ relative humidity. The four lines in the figure indicate the bit error rate at inner land, inner groove, outer land and outer groove, respectively. No change in the bit error rate is observed during a 2000-hour period. By using the same activation energy of $1.0 \mathrm{eV}$, which we have obtained for ordinary MO media, the lifetime of the new GIGAMO media is expected to duplicate those results and last at least 50 years under the condition of 25 degree Celsius.

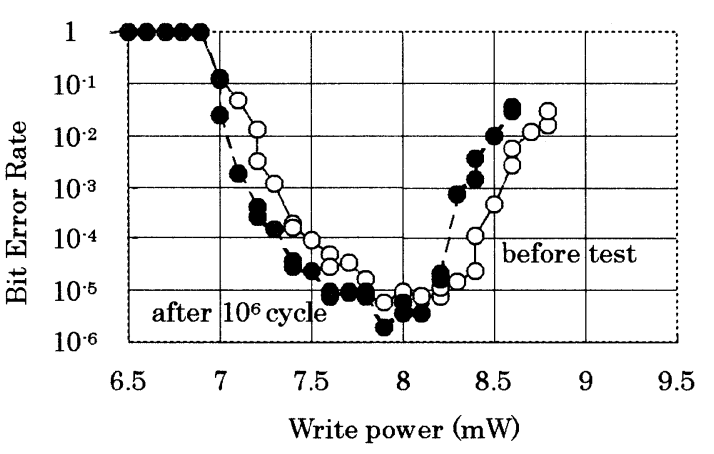

Fig. 9 Bit error rate as a function of write power for before and after cyclic erase, write, and read test of $10^{6}$ repetitions.

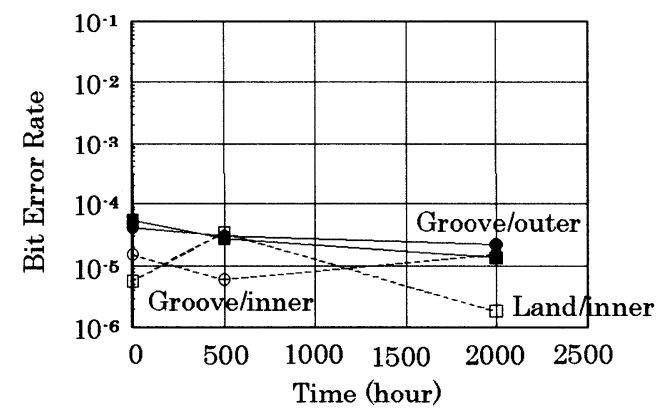

Fig. 10 Time dependence of bit error rate in accelerating life test at $80^{\circ} \mathrm{C}$ and $85 \%$ relative humidity.

\section{Conclusion}

By using the MSR technology and the land and groove recording method, a capacity of $2.3 \mathrm{~GB}$ per 3.5-inch disk is achieved while maintaining full downward compatibility. To make downward compatibility easier, a novel address format named Stagger ID is introduced. We ensure margins wide enough for practical use and high media reliability by evaluating the disks with the new GIGAMO drive. 


\section{References}

1) K. Itoh, H. Yoshimura, and K. Ogawa: Jpn. J. Appl. Phys., 39, $714(2000)$.

2) K. Matsumoto, K. Tamanoi, T. Tanaka, and K. Shono: Jpn. J. Appl. Phys., 35, L144 (1996).

3) T. Tanaka, M. Mihara, K. Tamanoi, T. Sugimoto, and K. Shono: IEEE Trans. Magn., 35, 580 (1999).

4) K. Tamanoi, T. Tanaka, T. Sugimoto, K. Matsumoto and K. Shono: Jpn. J. Appl. Phys., 36, L1104 (1997).

(Received February 18, 2002; Accepted June 20, 2002) 